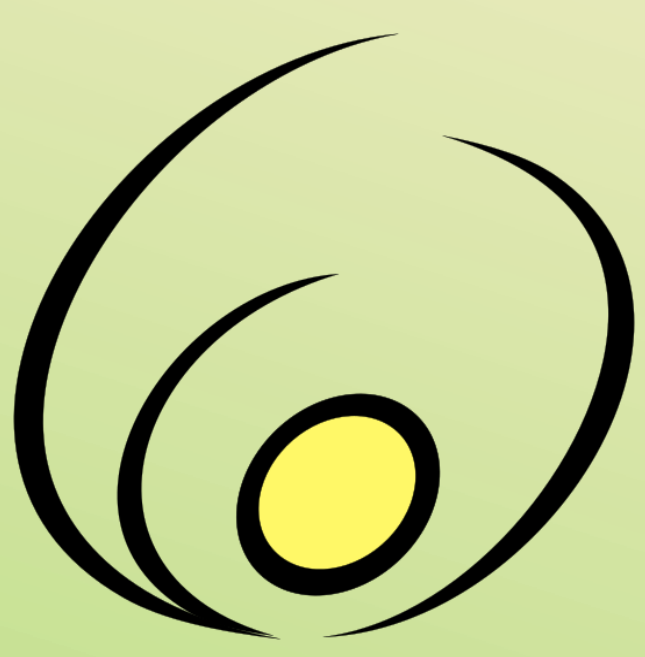

Fórum de

\section{Pró-Reitores}

\section{de Extensão}

das Instituições

Públicas de

\section{Educação Superior}

\section{Brasileiras}

Open access $\delta$ free available online

Revista Brasileira de Extensão Universitária

v. 8, n.2, p. 91-102 mai.- ago. 2017 e-ISSN 2358-0399

DOI: https://doi.org/10.24317/2358-0399.2017v8i2.4979

\title{
Memória e divulgação: ações educacionais da Casa da Ciência do Hemocentro de Ribeirão Preto na difusão do conhecimento
}

Fernando Rossi Trigo ${ }^{1}$, Flávia Fulukava do Prado², André Perticarrari ${ }^{3}$, Marisa Ramos Barbieri ${ }^{4}$

Resumo: A Casa da Ciência (CASA) nasceu e cresceu dentro de um centro de assistência e pesquisa da Universidade de São Paulo, o Hemocentro do Hospital das Clínicas da Faculdade de Medicina de Ribeirão Preto-SP. Em mais de 16 anos de atividade, pôde eleger difusão e divulgação científica como parte da sua ação metodológica, que se consolida a partir de uma trajetória baseada no registro e construção de uma memória educacional, focada na avaliação dos resultados, divulgados em diferentes formatos. Dentro do objetivo de difusão e divulgação científica, a CASA desenvolve programas regulares para alunos, professores e pesquisadores (Adote um Cientista, Pequeno Cientista e Saltimbancos da Ciência); promove eventos pontuais (janeiro e julho com o Férias com Ciência, em outubro com a Semana Nacional de Ciência e Tecnologia e, em março, com a Semana Nacional do Cérebro); e divulga seus resultados em diferentes formatos, tanto impressos quanto digitais (Website, Folhetins, Jornal das Ciências, dentre outros). Todas as atividades descritas neste trabalho - que integram ensino, pesquisa e extensão - apresentam como características comuns: a presença do pesquisador colaborador, o grupo de alunos, o acompanhamento da equipe da CASA que realiza o registro das diferentes manifestações de aprendizagem dos alunos, e sua posterior avaliação, que inclui a divulgação dos achados. Os principais desafios e limitações para a realização das propostas também são apresentados, com a finalidade de compartilhar com outros centros de extensão a experiência vivenciada pela Casa da Ciência de Ribeirão Preto.

Palavras-chave: Ensino de Ciências, Educação não-formal, Avaliação da Aprendizagem, Extensão Universitária, Divulgação Científica.

Content shared under Creative Commons Attribution 3.0 Licence CC-BY

1 Mestrando em Biotecnologia pela Faculdade de Medicina de Ribeirão Preto (FMRP-USP); integrante da equipe da Casa da Ciência do Hemocentro de Ribeirão Preto. fernando r trigo@yahoo.com.br (autor para correspondência)

2 Graduada em Medicina Veterinária e Música; mestre em Medicina Veterinária; integrante da equipe da Casa da Ciência do Hemocentro de Ribeirão Preto. flaviafulu@yahoo.com.br

3 Professor do Instituto Federal de São Paulo - Câmpus São Paulo (IFSP-SP). aperticarrari@gmail.com

4 Coordenadora da Casa da Ciência do Hemocentro de Ribeirão Preto. marisarbarbieri@gmail.com 
Memory and divulgation: educational actions of knowledge dissemination from the "Casa da Ciência" from Blood Center of Ribeirão Preto

\begin{abstract}
The House of Science (Casa da Ciência - CASA) was born and expanded in a health care and research center of the University of São Paulo, the Regional Blood Center of the University Hospital of the Medical School of Ribeirão Preto, São Paulo State, Brazil. Throughout its 16 years of activity, it was able to define scientific diffusion and dissemination as part of its methodological action, which is consolidated from a trajectory based on the registers and construction of an educational memory, focused on the evaluation of results, which were disclosed in different ways. With the aim of divulging and disseminating Science, the House of Science: 1. develops regular programs for students, teachers and researchers (Adopt a Scientist, Small Scientist and Science Mountebanks); 2. organizes single events (in January and July with the Vacations with Science, in October in the National Week of Science and Technology of Brazil and in March, in the National Week of the Brain) 3. publishes its results in both printed and digital format (website, leaflets, Journal of Science, among others). All the activities described in this work - that integrate education, research, and extension - have common characteristics: the presence of the collaborating researcher, the group of students, the follow-up of the House of Science team that keeps records of the different manifestations of students' learning and their subsequent evaluation predicting also the disclosure of its findings. The main challenges and limitations to the propositions are also shown here, aiming at sharing with other extension centers the experience lived by the House of Science of Ribeirão Preto.
\end{abstract}

Keywords: Science Education, Non-formal Education, Learning Evaluation, College Extension, Science Dissemination.

Memoria y divulgación: acciones educacionales de la Casa de La Ciencia del Hemocentro de Ribeirão Preto en la difusión del conocimiento

Resumen: La Casa de la Ciencia (CASA) nació y creció en un centro de servicios e investigación en la Universidad de São Paulo, el Banco de Sangre del Hospital Universitario de la Facultad de Medicina de Ribeirão Preto, São Paulo, Brasil. En más de 16 años de actividad, pudo elegir la difusión y la divulgación científica como parte de su acción metodológica, que se consolida a partir de una trayectoria basada en el registro y en la construcción de una memoria educativa, centrada en la evaluación de los resultados, publicados en diferentes formatos. Dentro del objetivo de la difusión científica, la CASA desarrolla programas regulares para estudiantes, profesores e investigadores (Adopta un Científico, Pequeño Científico y Saltimbancos de la Ciencia); promueve eventos puntuales (en enero y julio con el Vacaciones con Ciencia, en octubre con la Semana Nacional de Ciencia y Tecnología y, en marzo, de la Semana Nacional del Cerebro) y publica sus resultados en diferentes formatos, tanto impreso como digital (página web, folletos, Revista de la Ciencias, y otros más). Todas las actividades descritas en el trabajo - que integran enseñanza, investigación y extensión - tienen características comunes: la presencia del investigador, el grupo de estudiantes, el seguimiento del equipo de la CASA que lleva a cabo el registro de las diferentes manifestaciones de aprendizaje de los estudiantes y su posterior evaluación, que prevé también la comunicación de las conclusiones. Los principales desafíos y limitaciones para la realización de las propuestas también son presentados, con el objetivo de compartir con otros centros de extensión la experiencia vivida por la Casa de la Ciencia de Ribeirão Preto.

Palabras-clave: Enseñanza de Ciencias, Educación no formal, Evaluación de Aprendizaje, Extensión Universitaria, Divulgación Científica.

\section{Introdução}

A Casa da Ciência (CASA) do Hemocentro de Ribeirão Preto - Hospital das Clínicas da Faculdade de Medicina da Universidade de São Paulo - iniciou suas atividades em 2001, integrada aos projetos educacionais do Centro de Terapia Celular/Centros de Pesquisa, Inovação e Difusão (CTC/CEPIDS), apoiados pela FAPESP, com o compromisso inicial de "estimular a cultura científica nas escolas". Nestes anos de atividades, a convivência em um centro de pesquisa possibilitou imprimir à CASA uma trajetória metodológica fundamentada no registro e avaliação dos achados. A consolidação da proposta exigiu um longo período exploratório para ajustar uma rotina de avaliação e disponibilização dos resultados, de forma a caracterizar o trabalho de investigação, incorporando exigências que compõem projetos e relatórios de pesquisa, avaliando e divulgando, periodicamente seus resultados, na medida do possível.

Durante o processo educacional em parceria com pesquisadores - maioria pós-graduandos, de diferentes departamentos da USP que são convidados a participar das atividades no Hemocentro -, a CASA pôde eleger 
difusão e divulgação como objetivos dos seus programas. Estas frentes - previstas em formatos e alcances diferentes - paulatinamente vêm se fortalecendo e revelando resultados importantes no âmbito do ensinoaprendizagem.

Dentro do objetivo de difusão e divulgação científica, a CASA desenvolve programas regulares para alunos, professores e pesquisadores (Adote um Cientista, Pequeno Cientista e Saltimbancos da Ciência); promove eventos pontuais (janeiro e julho com o Férias com Ciência, em outubro com a participação na Semana Nacional de Ciência e Tecnologia e, em março, com a Semana Nacional do Cérebro). Além disso, divulga seus resultados em diferentes formatos, tanto impressos quanto digitais (Site, Folhetins, Jornal das Ciências, dentre outros). Estas propostas apresentam características em comum, ilustradas na Figura 1.

Este trabalho pretende descrever os programas da Casa da Ciência que integram, em suas diversas atividades, ensino, pesquisa e extensão, sendo a avaliação da manifestação dos alunos e o registro das atividades, pontos centrais para a divulgação do conhecimento.

\section{Ação metodológica da Casa da Ciência}

\section{Memória e Avaliação do Processo Educacional}

"A memória faz a ciência avançar": este é um dos pressupostos essenciais da CASA. Em sua ação metodológica procura-se articular três fases, que não são exatamente sequenciais, mas que ocorrem quase simultaneamente: ensino/aprendizagem (programas regulares e pontuais); registro e avaliação (investigação) e difusão e divulgação em formatos variados (Figura 2).

A equipe da CASA trabalha para que os registros das atividades (filmagens, textos, desenhos, representações, documentários fotográficos, entre outros recursos) sejam realizados e organizados, para posterior consulta, avaliação e divulgação. Tais registros permitem que durante as reuniões regulares da equipe - sejam retomados alguns resultados e avaliados os programas em andamento. Assim, percebem-se pontos "fortes" e "fracos" do processo relacionado à comprovação da aprendizagem nas manifestações, principalmente nas perguntas realizadas durante os encontros com os especialistas. O que se percebe é que a presença de articulações complexas entre conteúdos, dúvidas que requerem a volta aos conceitos básicos e a construção de hipóteses ${ }^{\underline{1}}$. As análises permitem à equipe perceber a importância de conteúdos básicos para alcançar a construção de conceitos mais complexos (normalmente encontrados nos temas de pesquisa apresentados aos jovens) e a importância do exercício de articular conceitos, buscando alcançar níveis mais abstratos e complexos (VIANNA, 1982). Essa avaliação permite um feedback do que os jovens aprendem. Este é um dos momentos mais importantes, o da avaliação dos resultados dos programas, que usa o registro das atividades, captando os momentos mais significativos, o que define $\mathrm{o}$ andamento das propostas, possibilita redirecionamentos, conferindo às propostas certa flexibilidade. Atualmente, os registros constituem parte do acervo documental Ponto de Informação, Pesquisa $e$ Organização em Ciências - Pipoc (Figura 3).

\section{Divulgação dos achados}

A partir da consulta da memória educacional é possível avaliar os programas e divulgar parte dos resultados alcançados. Esta é uma dificuldade, pois além da organização geral, a equipe precisa ter a habilidade da escrita. Entretanto, definir o formato de divulgação mais adequado para cada categoria de resultados não é uma ação direta. Ao longo dos seus 16 anos de atuação, a CASA desenvolveu diferentes instrumentos e mecanismos de divulgação, alguns focados nas manifestações e diálogos dos alunos durante os encontros com especialistas, outros valorizando o conteúdo específico e atual que o pesquisador apresentava aos jovens, enquanto outros buscaram destacar as atividades e projetos, ou seja, as decisões presentes no plano de orientação dos grupos de investigação.

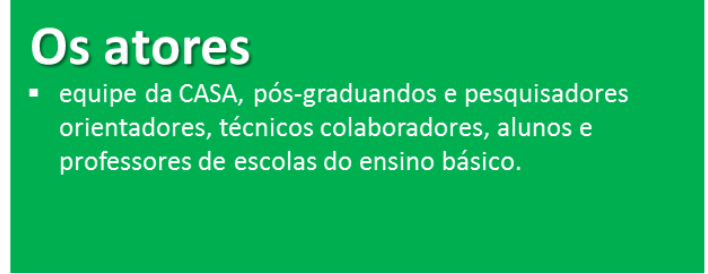

As atenções

focada na aprendizagem dos alunos (registros) e, a partir de 2012, na aprendizagem dos pós-graduandos que participam e orientam grupos de jovens em projetos de iniciação/alfabetização científica.

\section{As atividades}

- encontros organizados pela equipe que reúnem os alunos e pesquisadores em torno do conhecimento que é disponibilizado pelo especialista ou pelo aluno quando ensina seus pares.

\section{A divulgação}

- com base na avaliação dos resultados obtidos, são propostos diferentes formatos de divulgação.

Figura 1: Mesa demonstrativa e atividades lúdicas. Fonte: dos autores 

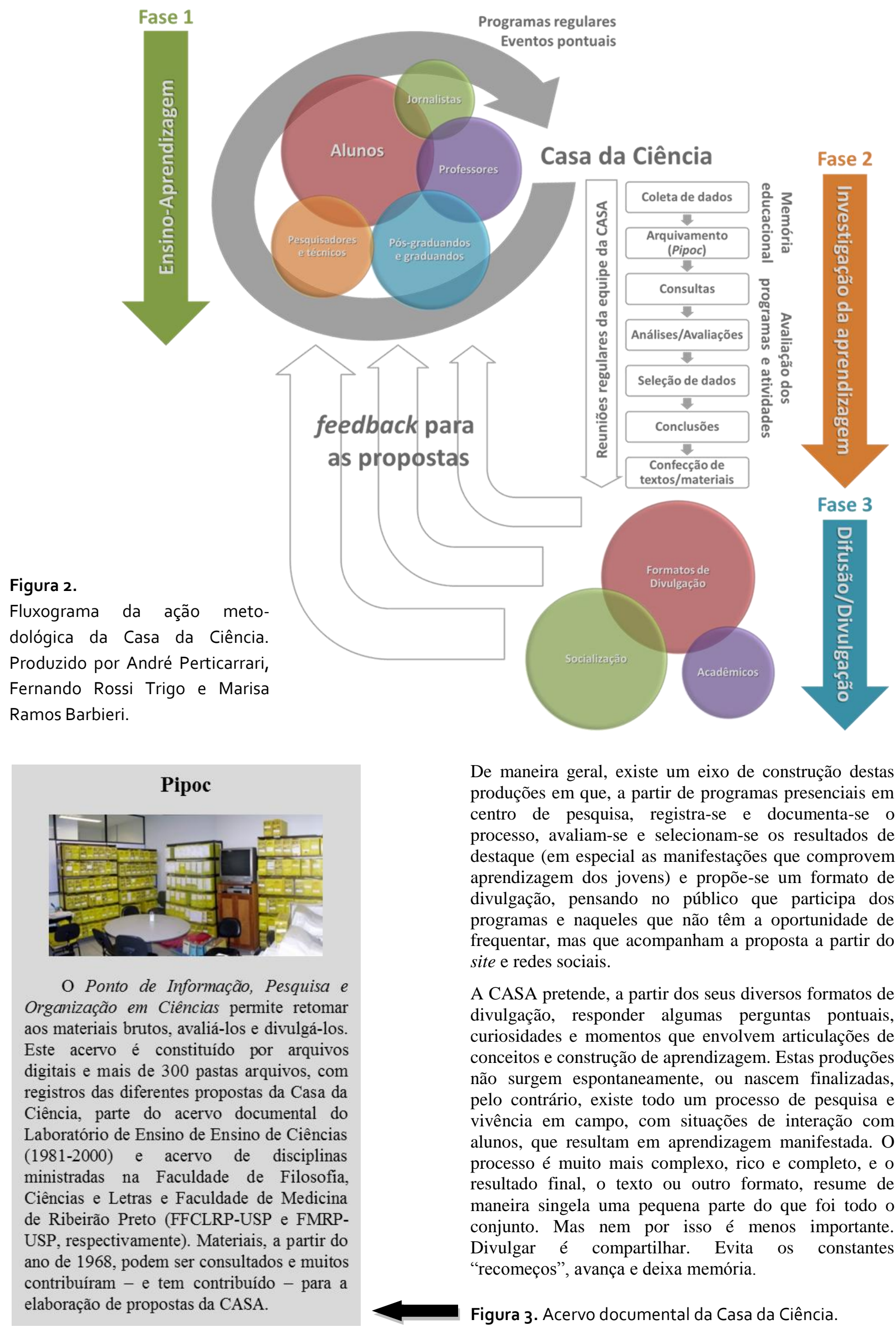

De maneira geral, existe um eixo de construção destas produções em que, a partir de programas presenciais em centro de pesquisa, registra-se e documenta-se o processo, avaliam-se e selecionam-se os resultados de destaque (em especial as manifestações que comprovem aprendizagem dos jovens) e propõe-se um formato de divulgação, pensando no público que participa dos programas e naqueles que não têm a oportunidade de frequentar, mas que acompanham a proposta a partir do site e redes sociais.

A CASA pretende, a partir dos seus diversos formatos de divulgação, responder algumas perguntas pontuais, curiosidades e momentos que envolvem articulações de conceitos e construção de aprendizagem. Estas produções não surgem espontaneamente, ou nascem finalizadas, pelo contrário, existe todo um processo de pesquisa e vivência em campo, com situações de interação com alunos, que resultam em aprendizagem manifestada. $\mathrm{O}$ processo é muito mais complexo, rico e completo, e o resultado final, o texto ou outro formato, resume de maneira singela uma pequena parte do que foi todo o conjunto. Mas nem por isso é menos importante. Divulgar é compartilhar. Evita os constantes "recomeços", avança e deixa memória.

Figura 3. Acervo documental da Casa da Ciência. 
Incorporação da divulgação na rotina de trabalho

A ação metodológica da CASA se estruturou principalmente a partir dos resultados com os alunos e pesquisadores. Sempre com o olhar nos jovens e nas suas diferentes manifestações, paulatinamente a equipe alcançou uma rotina de trabalho capaz de alcançar a divulgação de parte dos resultados de forma sistematizada.

Nestes anos de trabalho, algumas hipóteses foram comprovadas, como o enorme potencial do aluno em aprender conceitos complexos, próprios da pesquisa, e do pós-graduando em orientar jovens, melhorando sua formação e antecipando uma ação/prática que poderá ser solicitada futuramente em sua carreira acadêmica. A participação do pesquisador é constante, os jovens são orientados e tem a oportunidade de se aproximar da pesquisa, passam a fazer mais perguntas e articulam os conceitos, ou seja, estudam fazendo perguntas, além do desenvolvimento de habilidades como observar, anotar, levantar hipóteses (PERTICARRARI, et al., 2007; PERTICARRARI, TRIGO e BARBIERI, 2014). Os professores parceiros, que acompanham seus alunos, têm a oportunidade de atualizar e levar temas recentes para suas salas de aula.

A cada ano a equipe da CASA avalia os resultados alcançados em cada um de seus programas e promove mudanças nas diferentes propostas (Figura 2). No entanto, consolidar uma rotina de programas, orientada por diversas frentes, que inclui propostas regulares e atividades pontuais, corresponde a um desafio constante para a equipe. Articular informações dos alunos que frequentam a CASA, juntamente com os pesquisadores parceiros e professores regulares, registrando as frequências, as atividades desenvolvidas, alimentando os bancos de dados, os questionários aplicados, as fotos e filmagens dos encontros, os registros em cadernos de campo, a emissão dos certificados de participação, os resultados dos grupos de pesquisa, tudo isso focado na avaliação para a divulgação dos achados, valorizando-se a memória do processo educacional e não somente o recorte final do processo, constitui uma ação necessária, mas trabalhosa. Isto confirma a importância de todos os sujeitos envolvidos no processo, desde que tenham como referência e apoio, centros de pesquisa e ensino (COUTO et al., 2014). A cada nova proposta, elementos são adicionados à rotina, que passa a crescer cada vez mais em complexidade, mas que vem garantindo sucesso ao processo.

Rede de colaboração: escolas e centros de pesquisa

Ao divulgar seus resultados, a CASA espera compartilhar com outros centros de pesquisa e extensão as suas dúvidas, reflexões e resultados alcançados para que outros possam compartilhar e promover uma rede de articulação na área educacional. Esse movimento possibilita encurtar caminhos para outros centros de pesquisa, estabelece vínculos com escolas participantes, além de alcançar públicos distantes, que mantém adesão aos materiais divulgados e interagem no espaço digital.

A sociedade, em particular as escolas - com seus jovens, professores e demais profissionais- tem sido beneficiada pela atuação conjunta das escolas e centro de pesquisa, principalmente quando se estabelecem relações de longo prazo, como alguns exemplos de parceria de mais de nove anos. O professor parceiro encontra no centro de pesquisa um espaço de atualização e se vê autor de um processo educacional. O centro de pesquisa, neste caso o Hemocentro de Ribeirão Preto/HC-RP, adquire relevância como centro de difusão e divulgação científica, tornando pública parte das suas pesquisas para a sociedade. Segundo Marandino (2009, p. 166) "é consensual a ideia de que a relação entre as duas instituições pode ser muito profícua se professores e educadores de museus estabelecerem uma comunicação efetiva e articularem suas atividades".

Parte da história dos programas e atividades pode ser consultada na produção intitulada "O Livro da Casa da Ciência” (Figura 4), disponível gratuitamente para download no site da CASA.
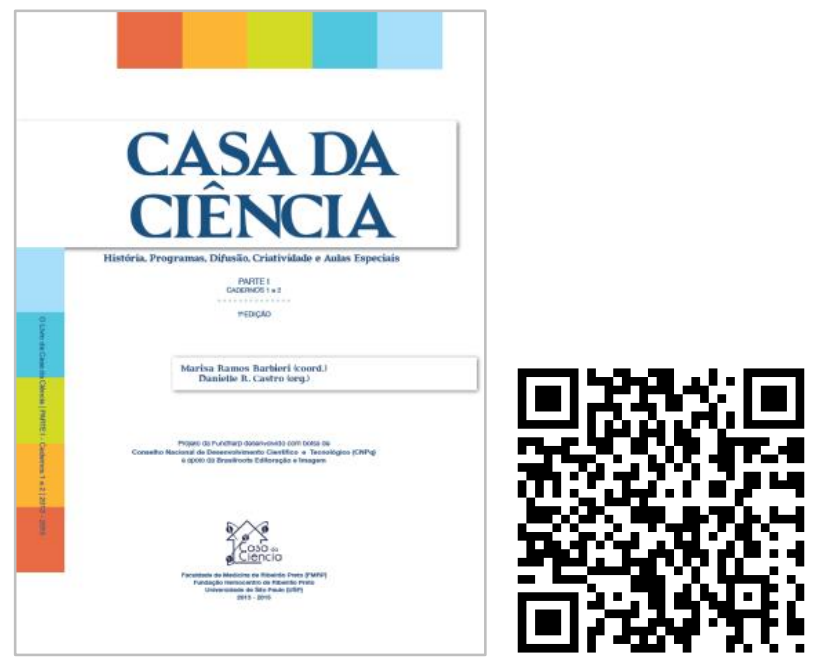

Figura 4. Projeto iniciado em 2012 e finalizado em 2015 por Danielle Castro, O Livro da Casa da Ciência é composto por 39 fascículos que contam parte da sua história.

\section{Descrição das experiências}

\section{Programas regulares}

\section{- Adote um cientista}

O programa educacional Adote um cientista, inicialmente chamado de Adote hoje o cientista de amanhã, iniciou-se no segundo semestre de 2005. Nasceu da experiência de projetos anteriores do Laboratório de Ensino de Ciências (LEC - décadas de 80 e 90) e de programas da Casa da 
Ciência, como o Caça-Talentos (2001-03) e o Fapesp Jr. (2004-05).

A denominação Adote um Cientista pressupõe a relação de cuidar e ser cuidado. Num sentido amplo, seria a ação de acompanhar e interagir. Em sua $12^{\mathrm{a}}$ edição, de caráter anual, passou por diferentes formatos, mas sempre se caracterizou por encontros semanais, com contato regular entre alunos do ensino básico (Tabela 1) e pesquisadores, através de palestras (Figura 5), de aproximadamente uma hora de duração, sobre temas atuais da pesquisa ${ }^{2}$.

\section{- Pequeno Cientista}

No Pequeno Cientista - idealizado em 2010, mas incorporado na rotina da CASA em 2012 -, alunos e pesquisadores participam de 10-11 encontros semanais (1 hora e 15 minutos de duração), em que desenvolvem pequenos projetos de investigação científica, com temas relacionados à sua pesquisa. A pergunta, a formulação de hipóteses, a observação, o registro em caderno de campo, a interpretação de resultados e a sua discussão articulada são trabalhados com os jovens.

Para a equipe da CASA, não existe idade para a iniciação científica, ou seja, quanto mais cedo o jovem se aproxima desta prática, maiores serão os desdobramentos para a sua formação, principalmente quando se enfatiza as questões próprias da pesquisa, como a articulação de conceitos básicos para a compreensão de questões complexas e a formulação de perguntas pertinentes à investigação.
Atualmente, o Pequeno Cientista - de caráter semestral encontra-se em sua $10^{\mathrm{a}}$ edição, com 115 projetos de orientação finalizados (Figura 6) e 16 projetos em andamento no primeiro semestre de 2017.

Ao final do semestre, os alunos realizam o Mural (Figura 7), evento em que os alunos apresentam seus achados e trocam experiências. Atualmente, encontra-se em sua $24^{\mathrm{a}}$ edição, com a divulgação dos resultados em formato de catálogos digitais (Figura 8), textos e vídeos publicados no site e nas redes sociais.

Tabela 1. Ao longo de mais de 10 anos de atividades, mais de 2.300 alunos de 24 cidades participaram do Adote um Cientista. Dados organizados por Luciana Silva, 2016.

\begin{tabular}{|c|c|c|c|}
\hline Ano & Alunos & Escolas & Cidades \\
\hline $\mathbf{2 0 0 5}$ & 250 & 54 & 7 \\
\hline $\mathbf{2 0 0 6}$ & 150 & 57 & 8 \\
\hline $\mathbf{2 0 0 7}$ & 164 & 40 & 5 \\
\hline $\mathbf{2 0 0 8}$ & 172 & 42 & 7 \\
\hline $\mathbf{2 0 0 9}$ & 196 & 32 & 5 \\
\hline $\mathbf{2 0 1 0}$ & 238 & 37 & 5 \\
\hline $\mathbf{2 0 1 1}$ & 231 & 27 & 4 \\
\hline $\mathbf{2 0 1 2}$ & 202 & 25 & 5 \\
\hline $\mathbf{2 0 1 3}$ & 235 & 29 & 6 \\
\hline $\mathbf{2 0 1 4}$ & 179 & 44 & 7 \\
\hline $\mathbf{2 0 1 5}$ & 170 & 27 & 7 \\
\hline $\mathbf{2 0 1 6}$ & 162 & 20 & 6 \\
\hline
\end{tabular}
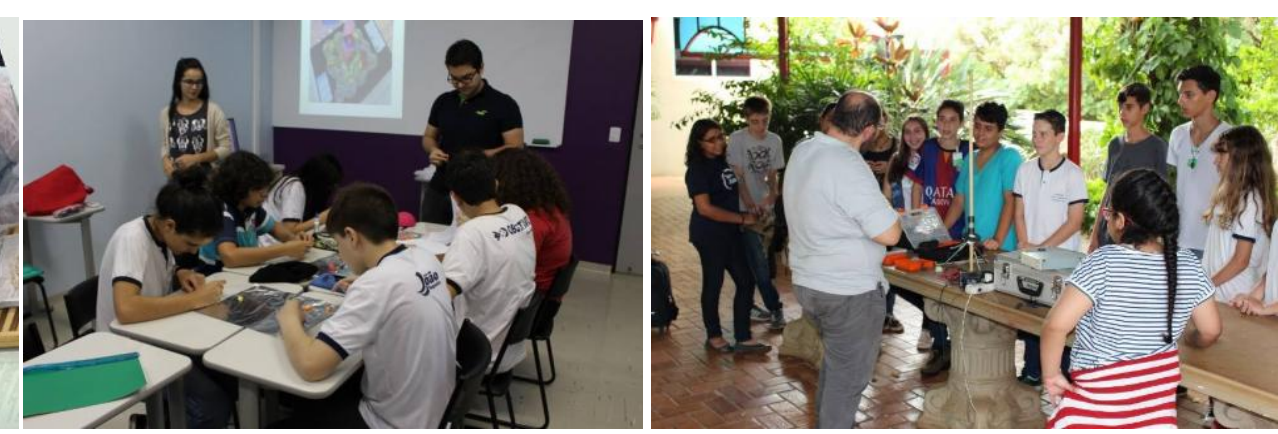

Figura 6. No Pequeno Cientista, pesquisadores orientam grupos de alunos em projetos normalmente relacionados às suas pesquisas. "Transformando plantas em medicamentos" (A) de Cláudia Regina de Souza, lara Baldim e Marcelo Martinez; "Detetives do DNA" (B) de Alexander Ferreira e Juliana Maçonetto; "Eletromagnetismo" (C) de Vinicius Godoi, foram alguns temas abordados.
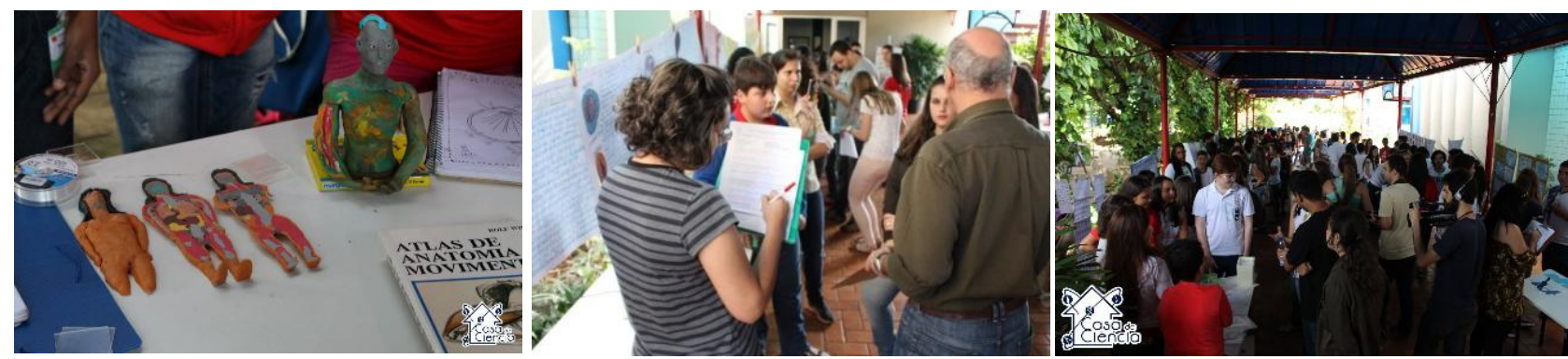

Figura 7. No Mural, os jovens têm a oportunidade de serem avaliados por diferentes pesquisadores e de aprenderem sobre os outros projetos de orientação. 


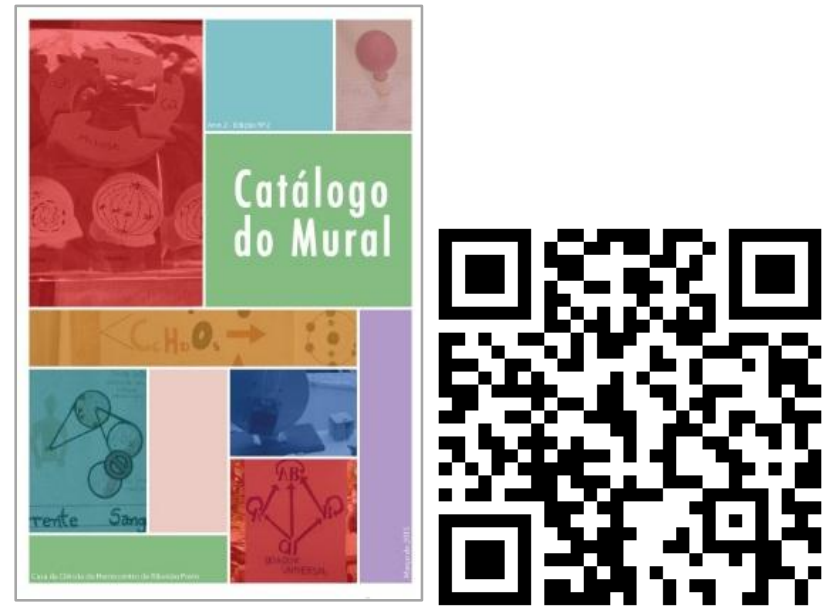

Figura 8. A proposta do Catálogo do Mural é divulgar momentos de aprendizagem de alunos, juntamente com relatos dos pesquisadores participantes. Utilize o código QR para conhecer essa produção

\section{- Saltimbancos da Ciência}

Inaugurado no segundo semestre de 2013, a proposta do Saltimbancos da Ciência é desenvolver projetos de investigação nas escolas, sob a orientação dos professores parceiros (Figura 9). Desta forma, a decisão do tema de estudo é realizada pelo professor, em parceria com os alunos. Os temas desenvolvidos normalmente refletem a realidade do ambiente escolar, alcançando resultados diferentes dos obtidos no Pequeno Cientista. A equipe da CASA, juntamente com os professores e pesquisadores (Figura 10), orienta e articula os achados, sempre com discussões em grupos, registro e organização de materiais para divulgação das propostas.

\section{Programas pontuais}

- Férias com ciência

Iniciado em 2010, a proposta é oferecer uma atividade 'leve' - adequada para as férias - para alunos de diferentes graus de ensino, incluindo universitários. Realizado no Museu e Laboratório de Ensino de Ciências-MuLEC (Figura 11), a cada evento um tema é selecionado e apresentado aos alunos em diversos formatos: projetos de investigação, bancadas de experimentos, palestras, atividades de campo, vídeos, oficinas de redação e HQs, dentre outros (Figura 12).

- Semana Nacional de Ciência e Tecnologia

A Semana Nacional de Ciência e Tecnologia (SNCT) é um evento temático proposto anualmente pelo Ministério da Ciência, Tecnologia e Inovação e pelo Conselho Nacional de Desenvolvimento Científico e Tecnológico para estimular a divulgação da pesquisa e a vocação de futuros cientistas. A CASA participa desde a primeira SNCT, em 2004, com exposições, palestras e atividades, tendo recebido mais de 10 mil visitantes ao longo das edições (Figura 13).
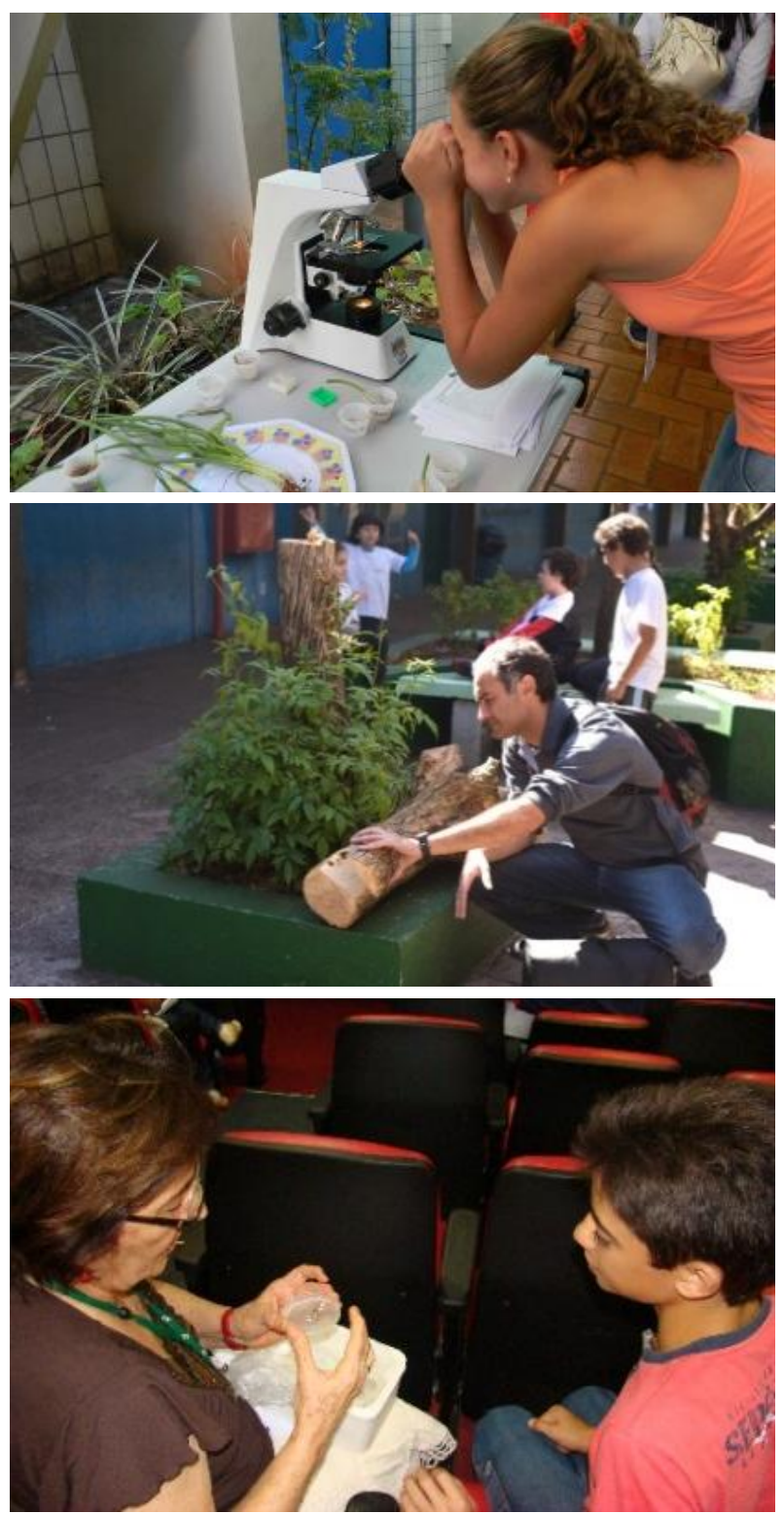

Figura 9. O Saltimbancos da Ciência revela o interesse dos jovens para questões do cotidiano. No segundo semestre de 2013, foram desenvolvidos 10 projetos e, no primeiro semestre de 2014, 14 projetos. Investigações sobre o crescimento das cebolinhas em diferentes condições ambientais (A), o uso de ninhos armadilhas para o estudo de abelhas solitárias (B) e o desvendamento do mundo dos besouros aquáticos (C), foram alguns dos temas explorados pelos jovens.

- Semana Nacional do Cérebro

Com o objetivo de divulgar e conscientizar o público sobre os progressos e benefícios da pesquisa em neurociências, a Sociedade Brasileira de Neurociências e Comportamento promove no Brasil, a Semana Nacional do Cérebro-SNC (Figura 14). Este evento é parte integrante da Brain Awareness Week (BAW), uma iniciativa internacional de divulgação das neurociências promovida pela Dana Alliance for Brain Initiatives, na qual a CASA participa desde 2011. 


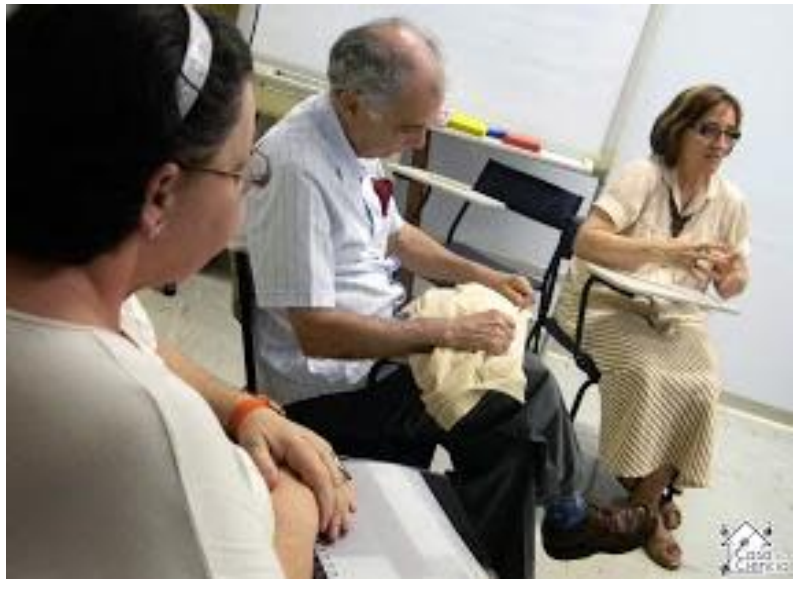

Figura 10. O professor, juntamente com seus alunos, é assessorado nos encontros pela equipe da CASA, que conta com a colaboração de pesquisadores parceiros para orientar os grupos escolares. Na ocasião, o prof. Dr. Marco Barbieri e a profa. Marisa Ramos Barbieri orientam as professoras em projeto de "Desenvolvimento da Criança e do Adolescente".

\section{Formatos de divulgação}

\section{- Site da Casa da Ciência}

Com o objetivo de divulgar os resultados alcançados pelos programas educacionais, fornecendo conteúdo exclusivo que colabore com a formação de professores e alunos, o site da CASA valoriza a construção do conhecimento, da aula, da avaliação e do processo de aprendizagem.

Graças aos registros das atividades e programas, o site antigo (Figura 15) finalizou sua atividade em fevereiro de 2016, e contou com mais de 650 páginas ativas, composto por textos exclusivos, desenhos produzidos pelos alunos, notícias sobre ciência e educação, materiais para download, dentre outros.
Em março de 2016, o site foi reformulado por Roberto Sanchez, que propôs novos layouts e ferramentas ao portal. Nesta proposta ${ }^{\underline{3}}$, composta atualmente por 357 páginas ativas, mais de 88.957 visualizações foram contabilizadas no ano (consulta em 15/05/2017).

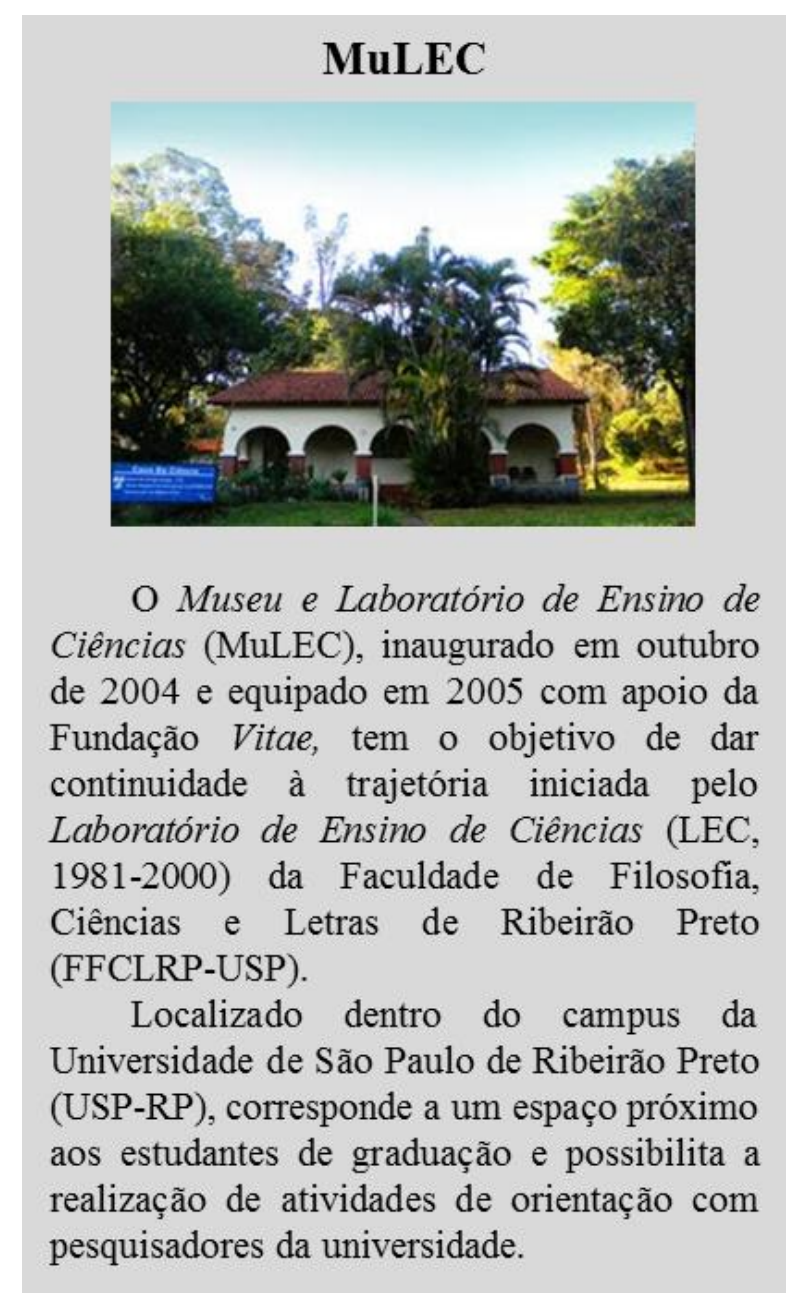

Figura 11: Museu e Laboratório de Ensino de Ciências.

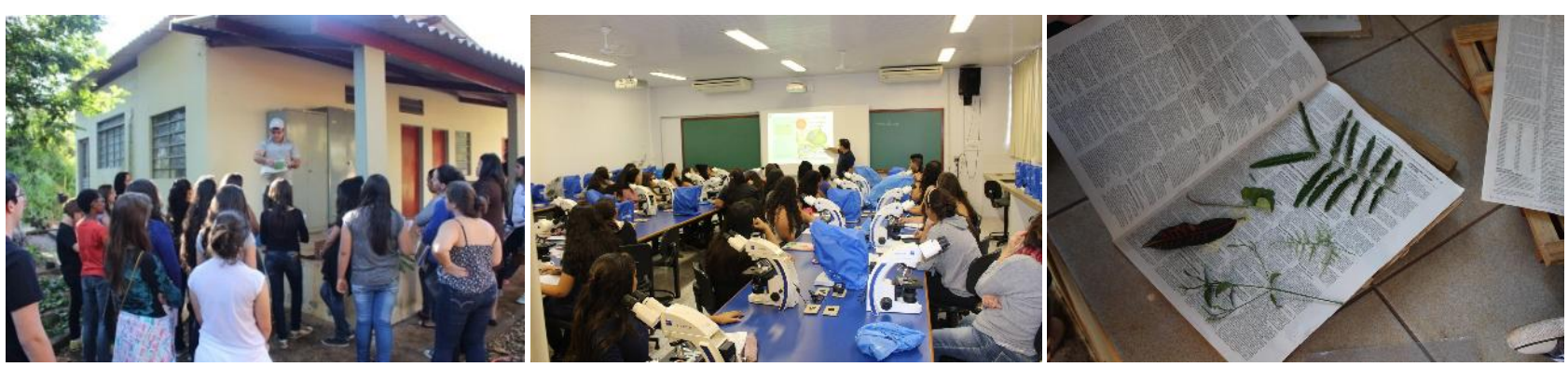

Figura 12. Em 2016, na sua $10^{a}$ edição, mais de 40 alunos do ensino básico participaram do Férias com Ciência, intitulado "Vegetando nas Férias". Orientados pelo prof. Dr. Milton Groppo Jr. da Faculdade de Filosofia, Ciências e Letras de Ribeirão Preto, os jovens participaram de atividades de campo, coleta, produção de herbário, montagem e observação de lâminas, dentre outras atividades, totalizando mais de 13 práticas em três tardes de evento. 


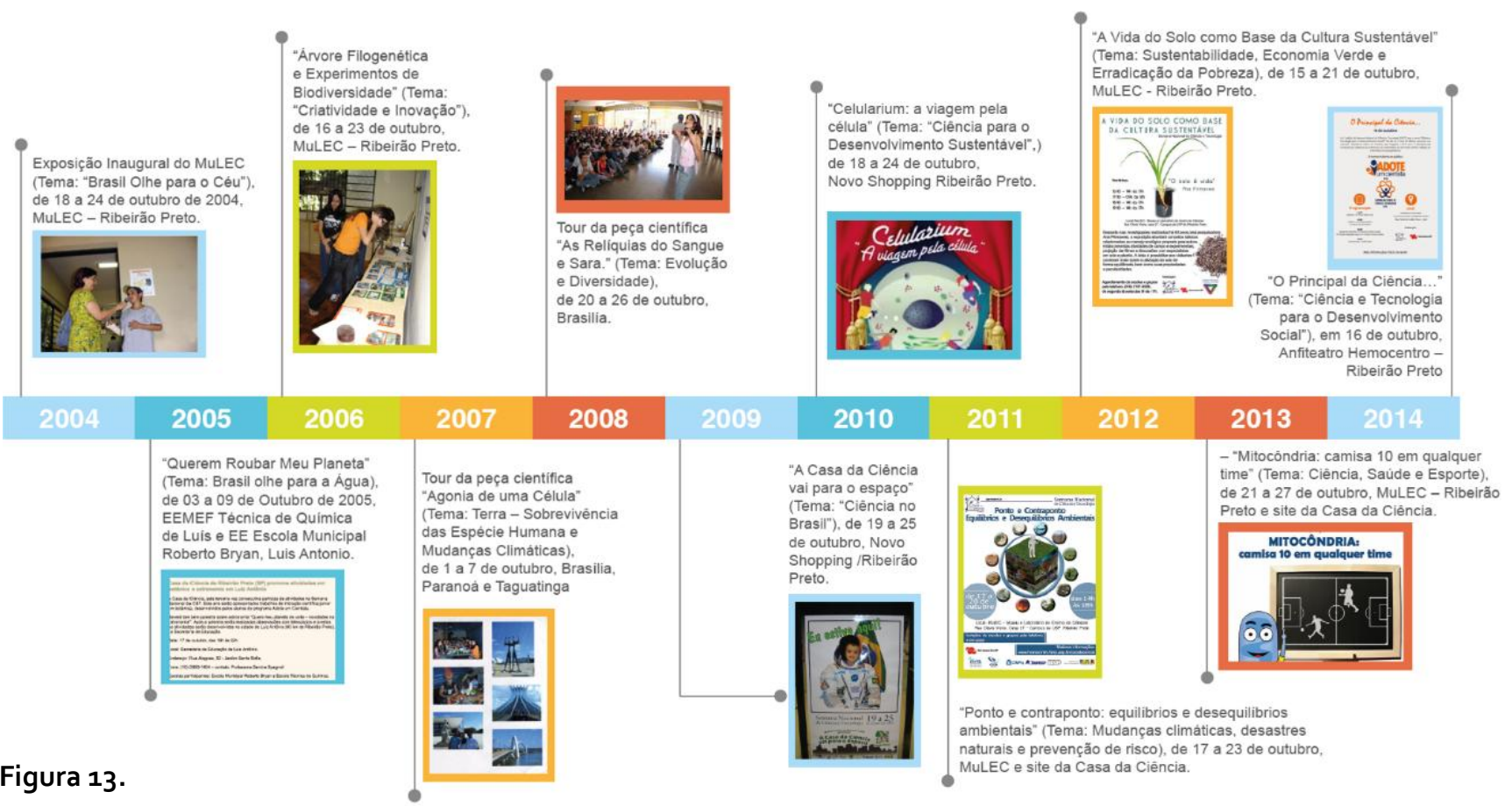

Participante em 13 edições da SNCT, a CASA sempre modificou a forma de se apresentar ao público. Em 2015, fez o lançamento digital do "O Livro da Casa da Ciência" e em 2016 abordou o tema Solo Grumoso, segundo referencial teórico 'Manejo ecológico do solo: a agricultura em regiões tropicais' (1981), da professora Dra. Ana Maria Primavesi.
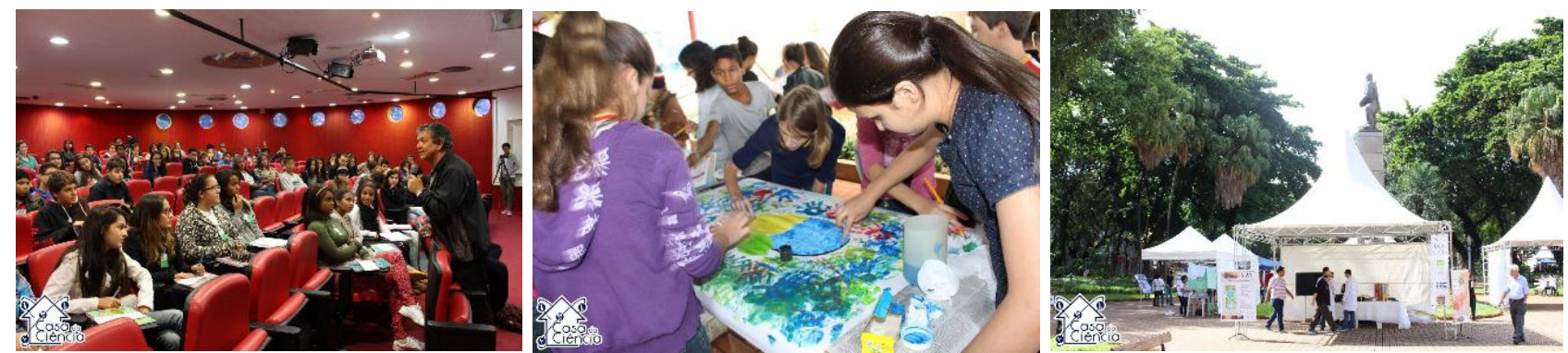

Figura 14. Em 2015, o prof. Dr. Norberto Garcia-Cairasco ministrou a palestra "Neurociência e Artes: da Arte nas Cavernas à Tecnologia Digital" e realizou com os alunos a "Oficina da Vinci". O resultado foi apresentado no dia 21 de março, durante o evento "SOS: O Cérebro na Praça", na Praça XV de Novembro, em Ribeirão Preto.

\subsection{5 cidades de 121 países}

\subsection{7 brasileiras}
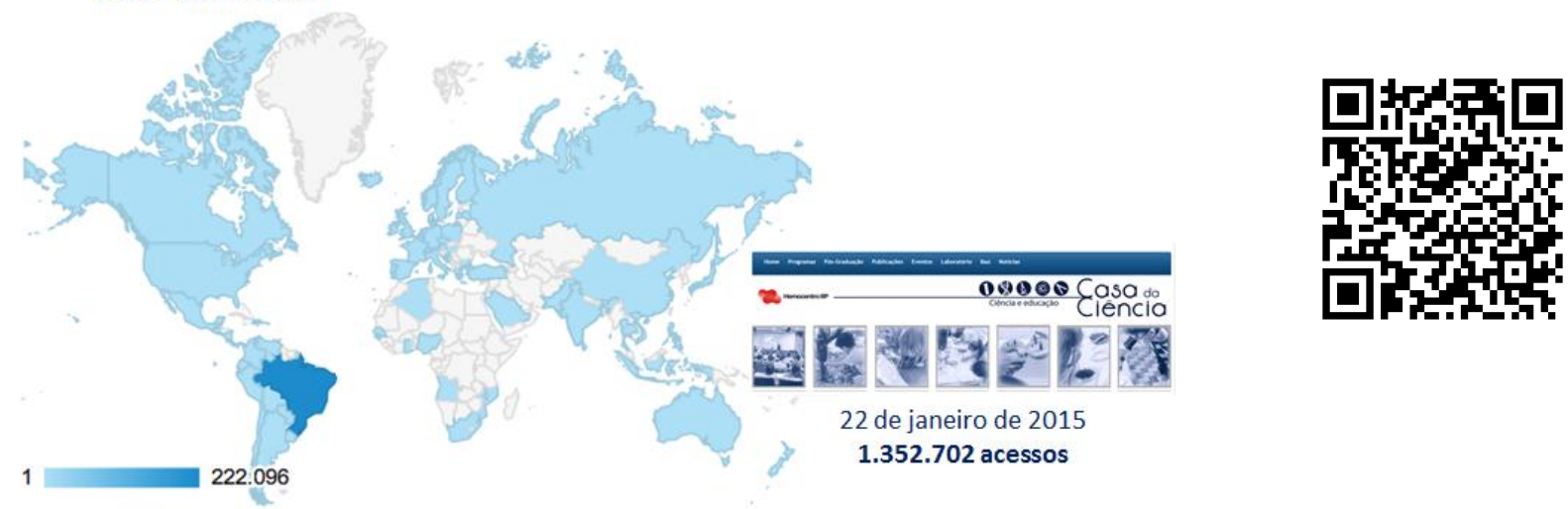

Figura 15. Em abril de 2014, o site alcançou a marca de um milhão de acessos, com a visitação de 2.565 cidades (1.097 brasileiras) de 121 países. Em janeiro do ano seguinte, a visitação totalizou mais de 1,3 milhões. Mapa gerado no Google Analytics por Gisele Oliveira. Utilize o código QR para acessá-lo. 
- Vídeos do Adote um Cientista e Pequeno Cientista

Em 2015, a CASA inseriu em sua rotina de trabalho a edição e divulgação das palestras do Adote um Cientista. Sob responsabilidade de Roberto Sanchez, integrante da equipe, as produções audiovisuais são editadas e disponibilizadas na íntegra no canal do You Tube da Casa da Ciência. Atualmente, o acervo possui 48 palestras, de aproximadamente 1 hora de duração, referentes as palestras do Adote um cientista e 38 vídeos dos grupos do Pequeno Cientista, com destaque aos depoimentos e conteúdos apresentados nos Murais. Outras produções, como o Adote uma Experiência com 24 vídeos, também estão disponibilizados (Figura 16).

Figura 16. No canal do You Tube da Casa da Ciência, 168 vídeos estão disponíveis. Data da consulta: 15/05/2017.

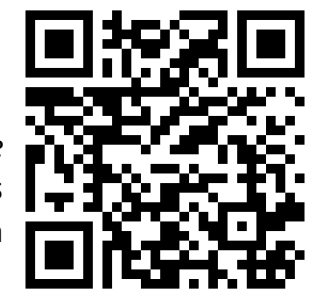

- Jornal das Ciências

O Jornal das Ciências (Figura 17), com 23 edições e uma edição especial, tem como característica básica a presença do pesquisador, tanto na produção quanto na revisão textual. A partir de um eixo temático determinado pela equipe, resultados obtidos nos diferentes programas e atividades da CASA são articulados, valorizando-se os conteúdos atuais, as interações e diálogos estabelecidos entre alunos e especialistas, possibilitando ao leitor a oportunidade de acompanhar o que se tem de mais novo nas discussões acadêmicas e os conhecimentos produzidos nas interações entre jovens estudantes e pesquisadores, muitos em processo de formação.

- Folhetins

Os Folhetins são textos produzidos por pesquisadores e especialistas, e tem como objetivo atualizar, articular e aprofundar temas específicos, que são diagramados em formato de folder. A proposta é trazer articulações inéditas que possam ser utilizadas em sala de aula (Figura 18).

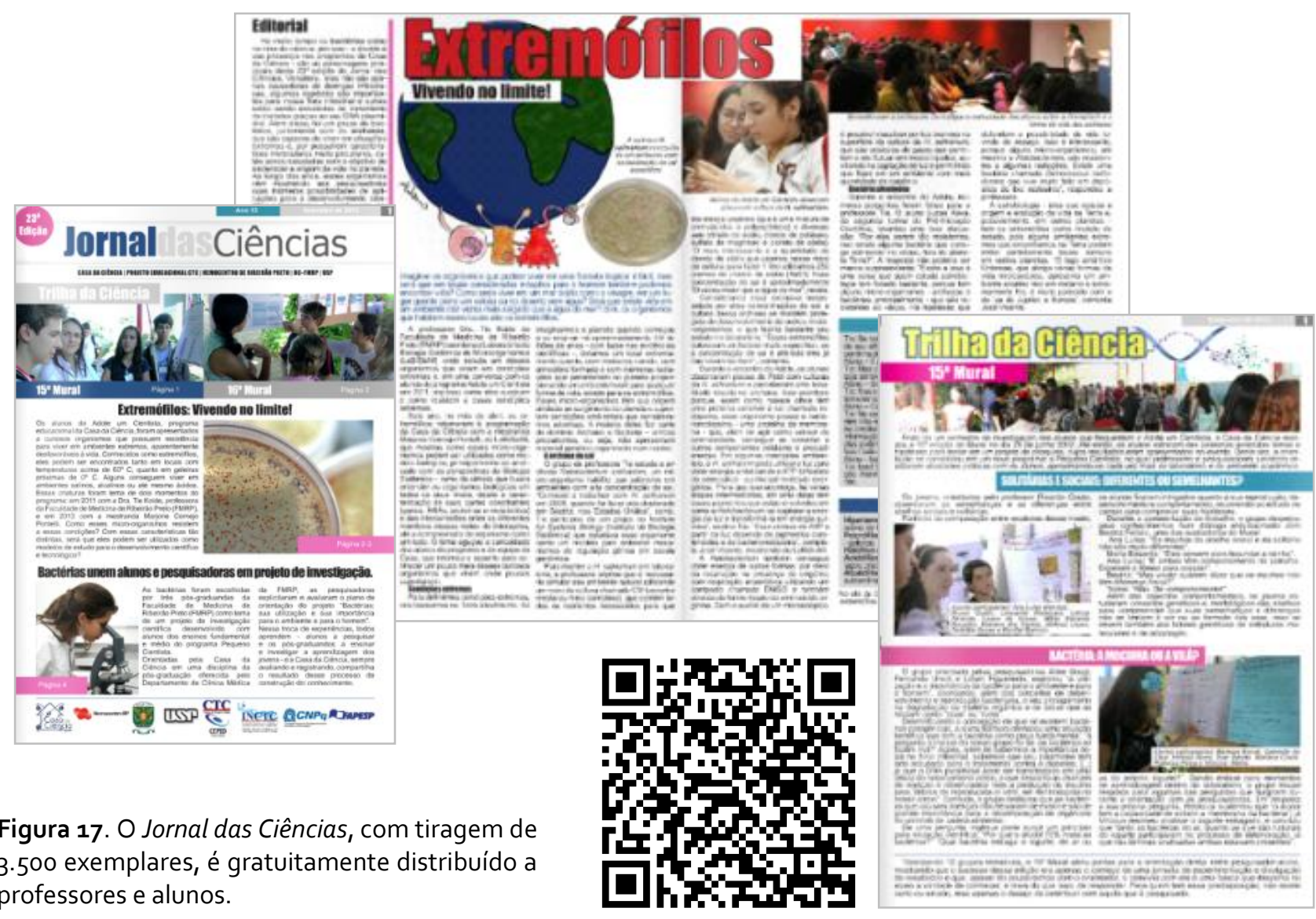




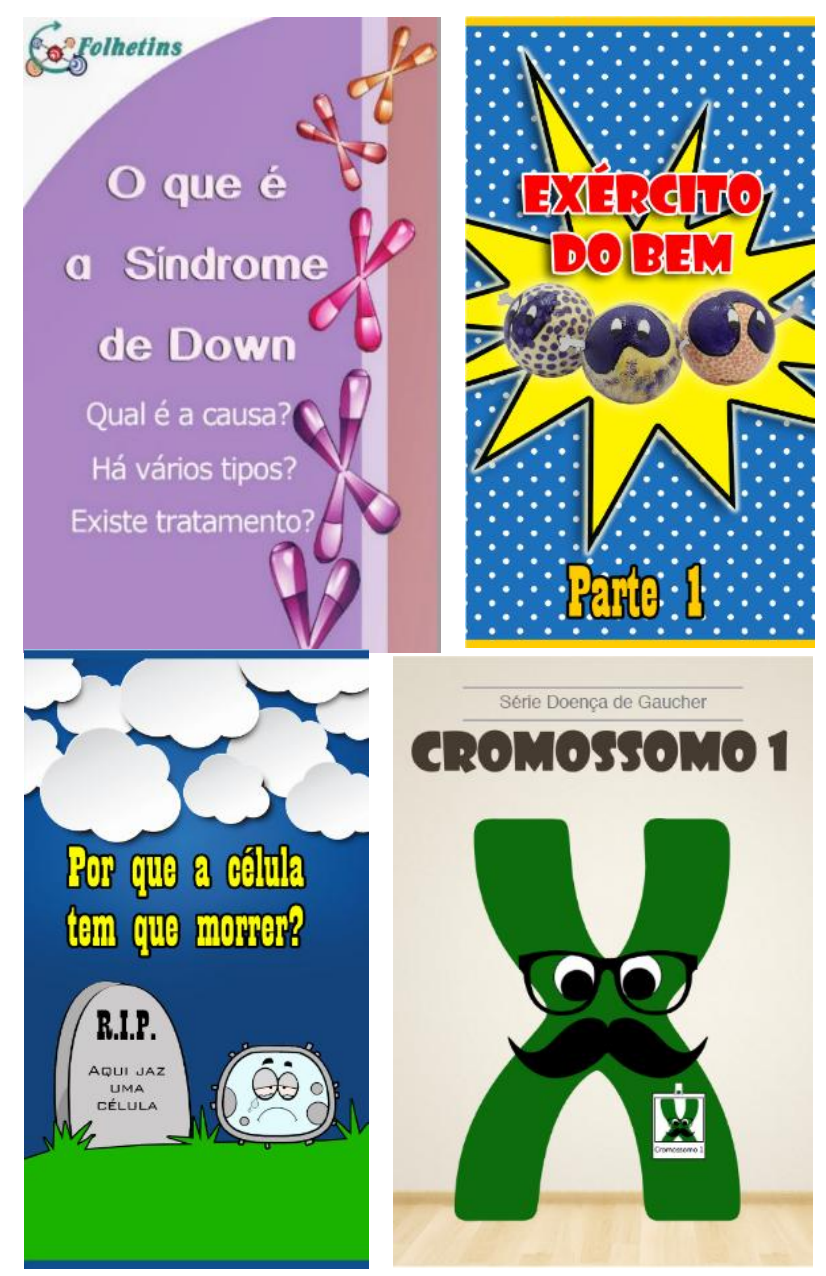

Figura 18. O primeiro Folhetim (2005) foi escrito e utilizado pelo médico Israel Gomes com o intuito de orientar, em centros de saúde, os pais de crianças com a síndrome de Down. As demais propostas foram produzidas a partir dos grupos de orientação da atividade Pequeno Cientista.

\section{Considerações finais}

Com 16 anos de história, a CASA tem proposto diferentes programas educacionais que aproximam alunos, professores de ensino básico e pesquisadores em centro de pesquisa. Entretanto, consolidar uma rotina de programas, orientada por diversas frentes, corresponde a um desafio constante para a equipe. As atividades de extensão, quando associadas ao registro e documentação, focados para a divulgação dos resultados alcançados nos diferentes programas educacionais, permitem alcançar e consolidar a memória do processo educacional (BARBIERI et al., 2001). A partir dele, a divulgação é facilitada, atingindo níveis mais complexos, pois, com a participação do pesquisador especialista na área, que produz e/ou avalia o material antes de divulgá-lo, garante-se credibilidade às produções. A adesão dos pesquisadores, pós-graduandos, técnicos colaboradores foi gradualmente aumentando nestes anos. Mesmo assim, a divulgação das atividades da CASA ainda está distante da ideal. Há o desejo de ampliar o número das atividades, principalmente por parte dos professores do ensino básico, que trazem seus alunos; porém, com uma equipe reduzida para tantas atividades, isso dificilmente seria possível. Os programas exigem: organização das atividades, contato com pesquisadores, orientações voltadas aos pós-graduandos, aos alunos, organização dos cronogramas e dos espaços para os encontros, controle de presença, filmagens, acompanhamento das atividades para captar momentos em caderno de anotações, elaboração de questionários, correções, escrita/redação de textos de divulgação, relatórios, artigos, reuniões, dentre outros. Pelo fato da equipe ser composta, em sua maioria, por bolsistas, portanto rotativos, os integrantes precisam de um certo tempo para compreender a forma de trabalho, identificar as manifestações, posturas e comportamentos, e as formas de escrever e divulgar os temas. Isso leva tempo, exige compreensão do método e fundamentos teóricos, para uma equipe que sempre se renova entrando novos bolsistas periodicamente. Ainda há a dificuldade na coleta de dados de aprendizagem pela equipe, e o desafio de acompanhar os grupos de orientação do Pequeno Cientista, que são descentralizados. A orientação dos pesquisadores na elaboração dos projetos e incentivo à redação e divulgação de textos da sua área de conhecimento para o site e demais veículos de divulgação ainda é muito singelo, com enorme potencial de sucesso, desde que incorporado à sua rotina de trabalho. Avaliar a aprendizagem dos alunos também corresponde a um grande desafio, não apenas para a equipe da CASA, mas para todos os participantes.

Contudo, apesar das dificuldades encontradas nos diferentes percursos das ações metodológicas da CASA, verifica-se que um caminho foi consolidado, tendo como ponto central a participação dos pesquisadores e especialistas, que detêm o conhecimento e dão aval aos instrumentos de divulgação de um lado e a participação das escolas de outro lado, cuja avaliação das manifestações dos alunos no contato com os pesquisadores - realizada pela equipe da CASA fornece a matéria prima para a divulgação científica. A parceria avaliada entre pesquisadores e alunos do ensino básico tornou-se a fonte do processo de divulgação realizada na Casa da Ciência, que procura integrar ensino, pesquisa e extensão nas atividades e programas que desenvolve.

\section{Agradecimentos}

Agradecemos a todos os profissionais e parceiros do Hemocentro de Ribeirão Preto e da Universidade de São Paulo que apoiaram e apoiam o projeto da Casa da Ciência, em especial os integrantes da equipe da CASA, Bárbara Naves, Gabriela Zaiuth, Lívia Pacheco, Luciana Silva e Roberto Sanchez; as jornalistas Danielle Castro e Gisele Oliveira; os parceiros Ricardo Couto e Vinicius Anelli. Agradecemos a todos professores parceiros e as prefeituras das cidades de Dumont, Luis Antonio e Cravinhos que apoiam o projeto. Agradecemos a colaboração de Lívia Pacheco para a produção do 
Abstract e de Fernanda Udinal para o Abstract e Resúmen. Agradecemos também as instituições de fomento Fundação de Amparo à Pesquisa do Estado de São Paulo (FAPESP) e Conselho Nacional de Desenvolvimento Científico e Tecnológico (CNPq) pelo apoio financeiro e institucional nesses anos de projeto.

\section{Notas}

1. Para exemplificar, em um dos encontros, os alunos discutiam sobre o complexo principal de histocompatibilidade (MHC I) e uma das alunas levantou uma questão sobre o Plasmodium sp (causador da Malária). Intrigou-lhe o fato de que a ausência do MHC I nas hemácias, que não têm núcleo quando circulantes, pode ser uma provável explicação da sua infecção por este parasita: "Será que o Plasmodium sp invade a hemácia porque ela não tem o MHC I?

2. Para exemplificar, em 2017, o professor Dr. Guilherme Lucas da Faculdade de Medicina de Ribeirão Preto (FMRP-USP) ministrou a palestra "O que vídeo games podem fazer com o nosso cérebro?”, trazendo aos alunos questões atuais da neurociência. Para conhecer outros temas abordados, acesse < $\underline{\text { www.casadaciencia.com.br }>\text {. }}$

3. Para conhecer a proposta, acesse < $\underline{\text { www.casadaciencia.com.br }>}$

\section{Contribuição de cada autor}

F. R. T., F. F. do P., A. P. e M. R. B. escreveram o texto final; F. R. T contribuiu com as análises estatísticas; F. R. T e M. R. B. planejaram o projeto, e M. R. B. atuou como coordenadora e orientadora dos bolsistas da equipe da Casa da Ciência.

\section{Referências}

BARBIERI, M. R.; SICCA, N. A. L; CARVALHO, C. P. A construção do conhecimento do professor: uma experiência de parceria entre professores do ensino fundamental e médio da rede pública e a universidade. Ribeirão Preto: Holos, 2001.

COUTO R.M; CASTRO, D.R.; TRIGO, F.R; PERTICARRARI, A.; BARBIERI, M.R. Professor e jornalista na escola: produção e divulgação de conhecimento. Raízes e Rumos. v. 2, n. 2, p. 75-84, 2014.
MARANDINO, M.; SELLES, S.E; FERREIRA, M.S. Ensino de biologia: histórias e práticas em diferentes espaços educativos. São Paulo: Cortez, 2009.

PERTICARRARI, A.; BARBIERI, M.R; GODOI, V.M.; COVAS, D.T.; AUGUSTO, A. Pós-graduandos e alunos do ensino básico em um trabalho de difusão científica. Experiências em Ensino de Ciências, v. 2, n. 1, p. 7989, 2007.

PERTICARRARI, A.; TRIGO, F.R.; BARBIERI, M.R. A parceria entre um centro de ciências e uma escola pública e sua implicação no processo de ensino e aprendizagem de ciências. Revista Brasileira de Ensino de Biologia, n. 7, p. 638-649, 2014.

PRIMAVESI, A. Manejo Ecológico do Solo: a agricultura em regiões tropicais. São Paulo: Nobel, $3^{\text {a }}$ ed., 1981.

VIANNA, H. M. Planejamento de Testes. In: VIANNA, H. M. Testes em Educação. 4a ed. São Paulo: IBRASA, 1982, p. 29-47.

\section{Como citar este artigo:}

TRIGO, F. R.; PRADO, F. F. DO; PERTICARRARI, A.; BARBIERI, M. R. Memória e divulgação: ações educacionais da Casa da Ciência do Hemocentro de Ribeirão Preto na difusão do conhecimento. Revista Brasileira de Extensão Universitária, v. 8, n. 2, p. 91-102, 2017. Disponível em: $<$ https://periodicos.uffs.edu.br/index.php/RBEU/article/view/ 4979/pdf $>$ 